\title{
Characterization of high-quality pseudomorphic InGaAs/GaAs quantum wells by luminescence and reflectance techniques
}

\author{
J. Pamulapati and P. Bhattacharya \\ Center for High-Frequency Microelectronics, Department of Electrical Engineering and Computer Science, \\ The University of Michigan, Ann Arbor, Michigan 48109-2122 \\ R. L. Tober \\ U.S. Army Laboratory Command, Harry Diamond Laboratories, 2800 Powder Mill Road, Adelphi, \\ Maryland 20783-1197
}

\begin{abstract}
J. P. Loehr and J. Singh
Center for High-Frequency Microelectronics, Department of Electrical Engineering and Computer Science, The University of Michigan, Ann Arbor, Michigan 48109-2122
\end{abstract}

(Received 14 June 1991; accepted for publication 24 January 1992)

\begin{abstract}
Reflectance and photoluminescence spectroscopy have been used to study the optical properties of high quality $\operatorname{In}_{x} \mathrm{Ga}_{1}-x \mathrm{As} / \mathrm{GaAs}(0.13 \leqslant x \leqslant 0.30)$ single quantum wells. The results show strong agreement with the theoretical model used taking into account the strain potential. The agreement of the theoretical model, though, deviates from the experimental results for large values of excess strain in the well. For the case of the large strain $(x=0.30)$ the reflectance indicates the strain in the well is hydrostatic rather than biaxial. The relevance of this fact is discussed in relation to device performance.
\end{abstract}

\section{INTRODUCTION}

Epitaxial growth techniques such as molecular beam epitaxy (MBE) and metalorganic chemical vapor deposition (MOCVD) routinely provide two-dimensional (2-D) electronic systems by allowing fabrication of quantum wells. The realization of quantum wells is possible because of the layer-by-layer growth mode that is achievable under certain growth conditions. Since the two-dimensional systems first proposed by Tsu and Esaki, ${ }^{1}$ the GaAs/AlGaAs system has been thoroughly studied. Increased emphasis recently, though, has been on the strained or pseudomorphic systems. The potential for these systems has been demonstrated both for use in optical components and in high-speed electrical components. With the increased use of strained layers in devices, careful consideration must be given to determine the extent to which a layer remains pseudomorphic and growth continues to occur in a twodimensional layer-by-layer mode, and two dimensional.

This paper reports the use of the piezoreflectance $(\mathrm{PzR})$ and photoreflectance $(\mathrm{PR})$ techniques as well as low-temperature photoluminescence (PL) to characterize isolated, strained $\operatorname{In}_{x} \mathrm{Ga}_{1}{ }_{x} \mathrm{As} / \mathrm{GaAs}$ single quantum well structures. The data obtained from the PzR, PR, and PL are accurately predicted using a theoretical model developed by us.

\section{THEORETICAL CONSIDERATIONS}

To model the valence band edge in quantum wells, it is essential to include the coupling between the heavy hole $(\mathrm{HH},|3 / 2, \pm 3 / 2\rangle)$ and light hole ( $\mathrm{LH},|3 / 2, \pm 1 / 2\rangle$ ) states; the split-off band can often be neglected. We treat the coupling with the $4 \times 4 \mathrm{k} \cdot \mathbf{p}$ Hamiltonian, ${ }^{2}$ taking the effects of strain into account through the deformation potential theory. In the total angular momentum basis, the strain contributes a splitting $\delta$ between the light and heavy hole diagonal elements. For $\mathrm{In}_{x} \mathrm{Ga}_{1-x}$ As grown on GaAs and $\mathrm{In}_{0.53+x} \mathrm{Ga}_{0.47-x}$ As grown on InP it is given (in $\mathrm{eV}$ ) by $\delta=-5.96 \epsilon$, where the lattice mismatch $\epsilon$ is related to the excess In composition $x$ by $\epsilon=-(0.07) x .^{3}$ This splitting reduces the off-diagonal mixing between the $\mathrm{HH}$ and LH states and changes the effective masses of the holes. We solve the valence band structure in the presence of strain by discretizing the Luttinger-Kohn equation and diagonalizing the resulting matrix. ${ }^{4}$

In 2-D lattice-matched quantum wells, there is a lifting of degeneracy between the heavy hole $(\mathrm{HH}-13 / 2$ $\pm 3 / 2\rangle)$ states and the light hole ( $\mathrm{LH}-|3 / 2 \pm 1 / 2\rangle)$ due to quantum confinement. In the presence of biaxial strain produced in a 2-D quantum well, additional splitting is produced by the shear component of strain. The net separation is then given by

$$
\Delta E_{\text {tot }}(\mathrm{HH}-\mathrm{LH})=\Delta E_{\mathrm{QC}}(\mathrm{HH}-\mathrm{LH})+\delta,
$$

where $\Delta E_{\mathrm{OC}}$ is the splitting due to quantum confinement and $\epsilon$ is the misfit. In the InGaAs/GaAs system, if we assume a conduction band discontinuity, $\Delta E_{c} / \Delta E_{g}$ $>0.60,{ }^{5}$ then the light hole state will be confined in the GaAs region. ${ }^{6,7}$ With this band alignment, we do not expect to see the light-hole transition in the PzR spectra due to the low overlap of the $n=1$ and the light hole. The theoretical model does not include the effect of strain on the exciton binding energy. The latter is assumed constant at approximately $8 \mathrm{meV} .^{8}$

\section{EXPERIMENTAL TECHNIQUES}

Molecular beam epitaxial growth of the heterostructures was carried out on (100) semi-insulating GaAs substrates. Growth was initiated with a 30-period undoped GaAs $(20 \AA) / \mathrm{Al}_{0.30} \mathrm{Ga}_{0.70}$ As $(20 \AA)$ superlattice buffer followed by $2000 \AA$ of GaAs. These layers were grown at 
TABLE I. Compositions of $\operatorname{In}_{x} \mathrm{Ga}_{1}-{ }_{x} \mathrm{As} / \mathrm{GaAs}$ single quantum well for the different samples characterized.

\begin{tabular}{lcc}
\hline \hline Sample & $x$ & $\begin{array}{c}L_{2} \\
(\AA)\end{array}$ \\
\hline UMV 262 & 0.13 & 85 \\
& 0.16 & 85 \\
UMV 277 & 0.18 & 85 \\
& 0.21 & 85 \\
& 0.26 & 85 \\
UMV 278 & 0.18 & 130 \\
& 0.21 & 130 \\
UMV 1068 & 0.26 & 130 \\
\hline \hline
\end{tabular}

$620^{\circ} \mathrm{C}$. This was followed by the growth of $\mathrm{In}_{x} \mathrm{Ga}_{1-x} \mathrm{As}$ $(0.13 \leqslant x \leqslant 0.3)$ at $520^{\circ} \mathrm{C}$, at a rate $\leqslant 1.0 \mu \mathrm{m} / \mathrm{h}$. After growth of the strained well, $1000 \AA$ of GaAs was grown to provide quantum confinement and to isolate the etch well. The thickness of the strained well was set at $85 \AA$, for sample UMV 262 and UMV 277 and $130 \AA$ for sample UMV 278. In each of these samples, a series of strained quantum wells was grown. Decreasing the In content in the well for each consecutive quantum well allows us to measure the luminescence from all of the wells as well as allowing reflectance spectroscopy. Table I lists the structural details of grown samples.

The piezoreflectance apparatus closely resembled that used elsewhere, ${ }^{9}$ and therefore, only relevant details will be presented here. Sample substrates were glued to lead-zirconate-titanate piezoelectric transducers. A $400 \mathrm{~Hz} 185$ $\mathrm{V}_{\mathrm{rms}}$ square wave applied to the transducer produced strains $\left(\leqslant 10^{-5}\right)$ sufficient to modulate the electronic structure of the double-barrier samples, yet small enough to ensure a linear relation to $\Delta R / R$. The temperature of the sample was maintained by the use of a liquid helium cryostat. The use of the cryostat allowed reflectance measurement of the samples from 295 to $8 \mathrm{~K}$. Radiation from a tungsten-halogen lamp was dispersed through a $1 / 4 \mathrm{~m}$ monochromator and focused to a $\sim 250 \mu \mathrm{m}$ spot on the sample. The modulated reflectance signal was measured using a silicon $p-i-n$ photodiode and lock-in amplifier, then normalized digitally to the dc reflectance. Low-temperature $(8 \mathrm{~K})$ photoluminescence $(\mathrm{PL})$ measurements were made with a 1-m Jarrell-Ash spectrometer to ascertain the optical quality of the quantum wells.

\section{RESULTS AND DISCUSSION}

The PL spectra from the single quantum wells are shown in Figs. 1(a) and 1(b). The spectra are very sharp and excitonic in nature. There are several important aspects in the data of Fig. 1. The linewidths, which range from 1.24 to $2.68 \mathrm{meV}$, are extremely narrow for the compositions and well widths indicated, and approach those measured in high-quality GaAs/AlGaAs quantum wells. ${ }^{10}$ The sharpness of the excitonic features indicate that the samples have very little interface roughness. We believe

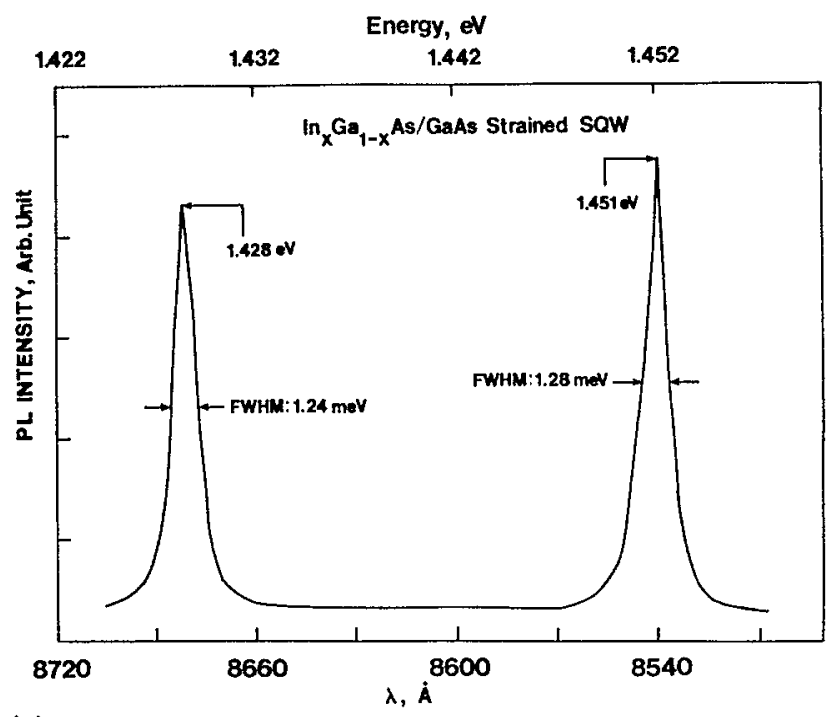

(a)

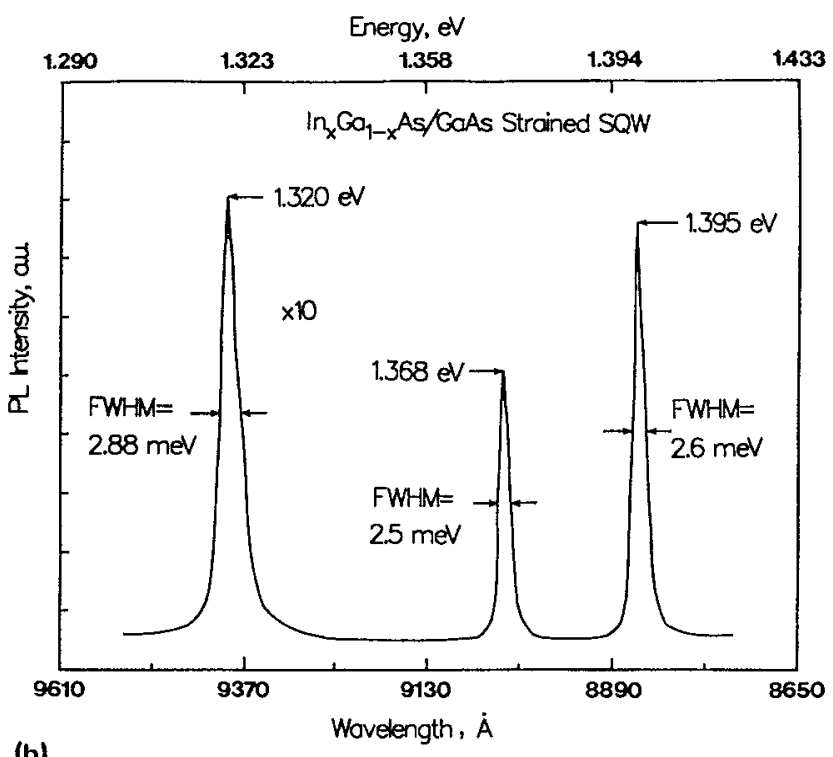

(b)

FIG. 1. Low-temperature (14 K) photoluminescence spectra of $\mathrm{In}_{x} \mathrm{Ga}_{1-x}$ As/GaAs strained SQW with (a) $L_{z}=85 \AA$ and (b) $L_{z}$ $=130 \AA$.

that the dramatic improvement in the optical quality results from (i) source and system preparation and purity, and (ii) the growth technique. With respect to the latter, the important factors are the incorporation of adequate buffers to trap impurities and dislocations, and smoothening of the growth front with an ideal growth rate and growth temperature.

Figures 2(a) and 2(b) show the PzR spectra obtained from UMV 262 and UMV 277, respectively. The two peaks with the largest magnitudes, in Fig. 2(a), are attributed to the $n=1-\mathrm{HH}$ transitions for the $\mathrm{In}_{0.13} \mathrm{Ga}_{0.87} \mathrm{As}$ and the $\mathrm{In}_{0.16} \mathrm{Ga}_{0.84}$ As pseudomorphic wells. The three largest transitions in Fig. 2(b) are attributed to the $n=1-\mathrm{HH}$ for the $\operatorname{In}_{x} \mathrm{Ga}_{1-x} \mathrm{As}(x=0.18,0.21,0.26)$. The higher energy transitions observed in the reflectance spectra are indicative of the second-order transitions. ${ }^{11,12}$ No light hole transitions are expected in this spectra due to the fact that 


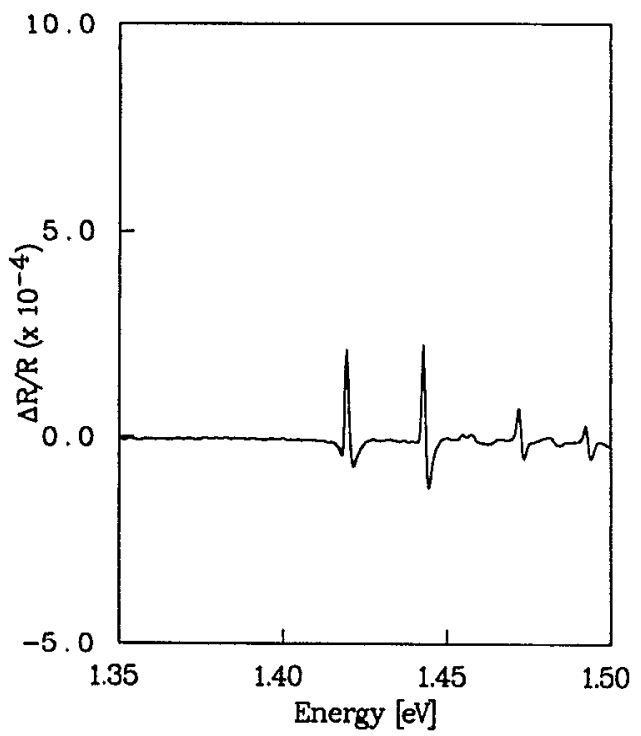

(a)

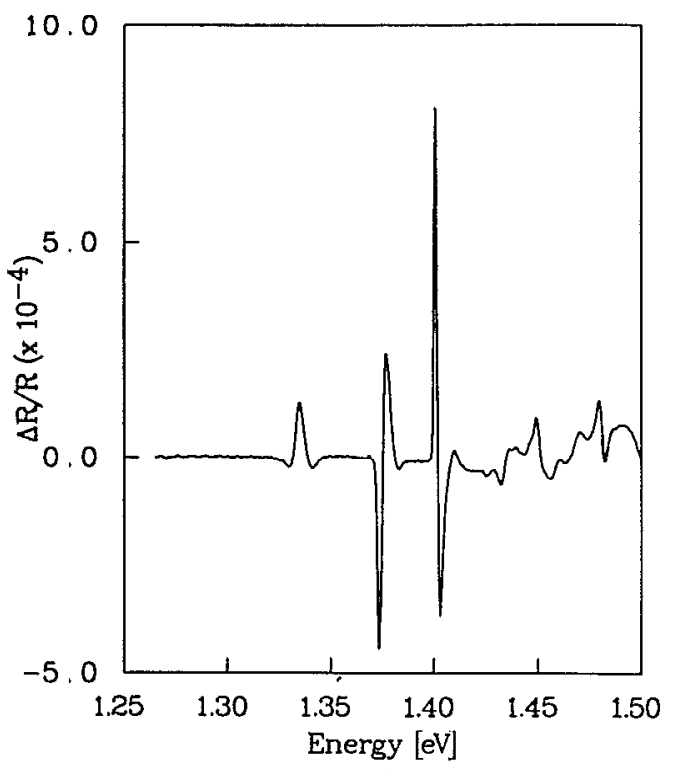

(b)

FIG. 2. Piezoreflectance spectra of samples with $85 \AA \operatorname{In}_{x} \mathrm{Ga}_{1}-{ }_{x} \mathrm{As}$ wells (a) $x=0.13$ and 0.16 , and (b) $x=0.18,0.21$, and 0.26 .

the light hole will be confined in the GaAs barriers ${ }^{6}$ and will have a very weak oscillator strength. The accuracy of these transitions is verified by a comparison with PL transitions and shows good agreement with theoretical predictions. Figure 3 shows the PzR spectra obtained from UMV 278. This sample is similar to UMV 277, with the exception of the well widths, which were set to $130 \AA$. From a comparison of Figs. 3 and 2(b) the energy dependence of the quantum well position with well width is evidenced. Utilizing these samples, good agreement is found between calculated and measured values of energy transitions. Figure 4 shows the fit between the experimentally measured transition energies and the theoretically predicted transition energies. As is evident in Fig. 4, the theoretical predictions closely match the measured values using a discon-

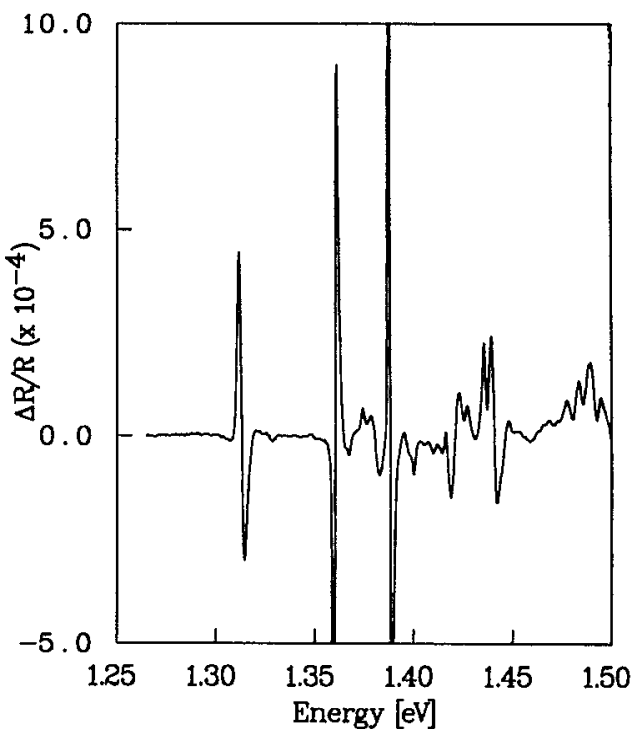

FIG. 3. Piezoreffectance spectrum of sample with $130 \AA \operatorname{In}_{x} G a_{1-x} A s$ wells with $x=0.18,0.21$, and 0.26 .

tinuity of 65:35. Equally evident in Fig. 4 is the small change in the theoretical model with changes in the band discontinuity factor.

Figure 5 shows PL spectrum from sample UMV 1068, which is a single quantum well with a $30 \AA \mathrm{In}_{0.3} \mathrm{Ga}_{0.7} \mathrm{As}$ well and $1500 \AA$ GaAs barriers. Figure 6 shows the PzR and photoreflectance (PR) spectra recorded from sample UMV 1068, at a sample temperature of $82 \mathrm{~K}$. From measurements on several calibration samples consisting of single layer GaAs, GaAs/AlGaAs QW, and InGaAs/GaAs $\mathrm{QW}$, we believe that the peaks labeled $\mathrm{A}$ and $\mathrm{B} / \mathrm{C}$ originate from $\mathrm{HH}$ and $\mathrm{LH}$ transitions, respectively. The peak labeled $\mathrm{D}$ originates from $\mathrm{GaAs}$ excitons and the features labeled $\mathrm{E}$ are Franz-Keldyish oscillations (FKOs). It is to be noted that the features labeled $A$ and $B$ are fairly sharp and well-defined. Since in this quantum well system we expect the light hole state to be confined in the GaAs, we

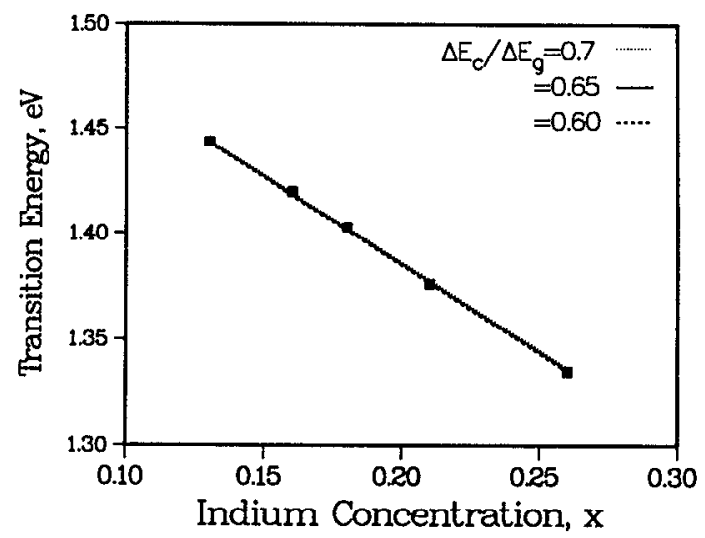

FIG. 4. Comparison of theoretically calculated and experimentally observed transition energies. The solid circles indicate the experimentally measured data and the solid line represents the calculated values. 


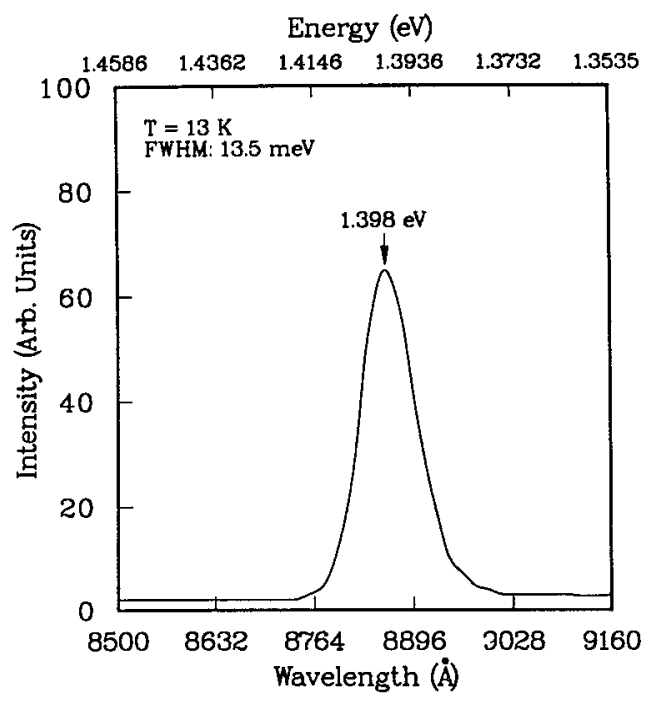

FIG. 5. Low-temperature photoluminescence spectra of $\mathrm{GaAs} / \mathrm{In}_{0.3} \mathrm{Ga}_{0.7} \mathrm{As} / \mathrm{GaAs}$ pseudomorphic heterostructure.

do not expect to see this transition. In the case of UMV 1068, shown in Fig. 6, though, if the InGaAs was surrounded by $\mathrm{GaAs}$ as would occur if the InGaAs regions were growing in 3-D islands, the strain on the InGaAs would primarily be of a hydrostatic nature. There is now clear evidence from our direct observation of such islands by in situ scanning tunneling microscopy (STM), ${ }^{13}$ and earlier work ${ }^{14}$ that island growth of strained layers under large biaxial strain does indeed lake place. The fact that we observe the LH resonance in sample UMV 1068 is also indirect evidence of such island growth. It is important to add that heterostructure interfaces using strained semiconductors, which grow in these modes, may be far from desirable and will have serious consequences on device performance. ${ }^{15}$ More study is needed with techniques such as migration enhanced epitaxy (MEE) to control these interfaces to prevent such degradation. During MEE growth the surface changes from cation stabilized to anion stabilized phase alternatively, corresponding to the opening of the source shutters. If the shutter opening time is properly adjusted, a complete recovery of the smoothness of the growth front is possible. Alternatively, if the growth can be controlled to produce uniform island formation, structures with three-dimensional quantum confinement, quantum dots, can be investigated.

\section{CONCLUSION}

The reflectance and photoluminescence results indicate the high quality of the single quantum well samples. The photoluminescence linewidths are among the narrowest for the well widths and compositions grown and are indicative of the sample purity and interface control. Furthermore, the transition energies of the experimental data are in excellent agreement with the theoretical model used to calculate the lransition energies. The study of these wells from the low In concentration range where the misfit is less than

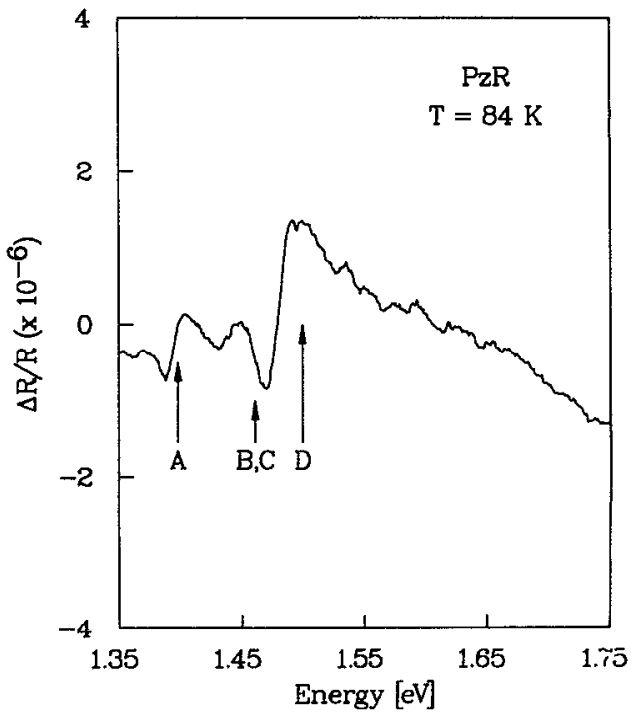

(a)

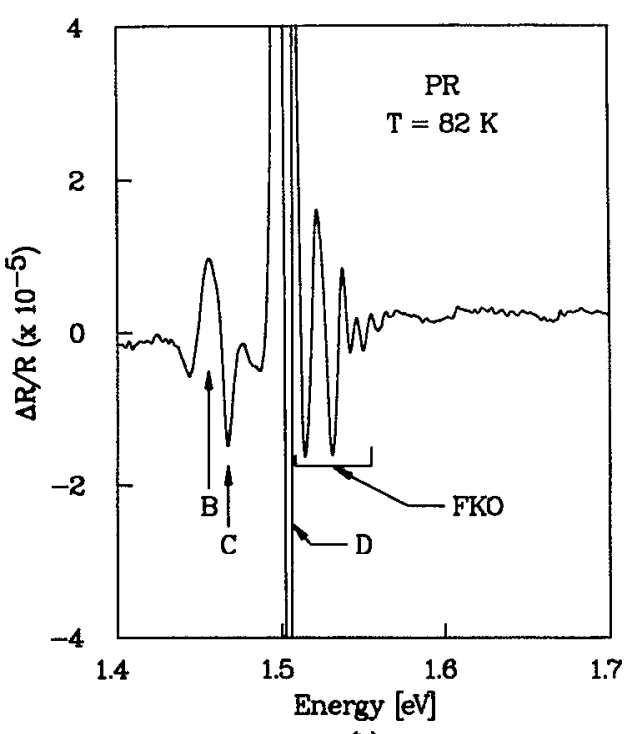

(b)

FIG. 6. Piezoreflectance (a) and photoreflectance (b) data measured at 84 and $82 \mathrm{~K}$, respectively, for the $\mathrm{GaAs} / \mathrm{In}_{0.3} \mathrm{Ga}_{0.7} \mathrm{As} / \mathrm{GaAs}$ pseudomorphic heterostructure.

$2 \%$ to the case where the misfit is greater than $2 \%$ leads to interesting results. When the excess strain exceeds $2 \%$ the layer no longer grows in a layer-by-layer mode, but prefers island formation. The latter leads to a strain in the layer which is hydrostatic in nature, and therefore results in features in the reflectance spectra which are unexpected. The changes in the nature of growth with excess In is extremely relevant for device fabrication and requires further study to control the growth.

\section{ACKNOWLEDGMENT}

The work was supported by the Army Research Office (URI program) under contract DAAL03-87-K0007.

${ }^{\text {I}}$ R. Tsu and L. Esaki, Appl. Phys. Lett. 22, 562 (1973). 
2J. M. Luttinger and W. Kohn, Phys. Rev. 97, 869 (1955).

${ }^{3}$ H. Kato, N. Iguchi, S. Chika, M. Nakayama, and N. Sano, J. Appl. Phys. 59, 588 (1986).

${ }^{4}$ M. Jaffe and J. Singh, J. Appl. Phys. 65, 338 (1989).

${ }^{5}$ N. Debbar, D. Biswas, and P. Bhattacharya, Phys. Rev. B 40, 1058 (1989).

'J. Y. Marzin, M. N. Charasse, and B. Sermage, Phys. Rev. B 31, 8298 (1985).

${ }^{7}$ Y. Zou, P. Grodzinski, E. P. Menu, W. G. Jeong, P. D. Dapkus, J. J. Alwan, and J. J. Coleman, Appl. Phys. Lett. 58, 601 (1991).

${ }^{8}$ G. Bastard and J. A. Brum, IEEE J. Quantum Flectron. QE-22, 1625 (1986).
${ }^{9}$ R. L. Tober, A. L. Smirl, T. F. Boggess, and J. N. Schulman, J. Appl. Phys. 64, 4678 (1988).

${ }^{10}$ J. Y. Marzin and J. M. Gérard, Superlatt. Microstructures 5, 51 (1989).

${ }^{11}$ G. D. Sanders and Y. C. Chang, Phys. Rev. B 35, 1300 (1987).

${ }^{12}$ J. P. Loehr and J. Singh, Phys. Rev. B 42, 7154 (1990).

${ }^{13}$ C. W. Snyder, D. Barlett, B. G. Orr, P. K. Bhattacharya, and J. Singh, J. Vac. Sci. Technol. B (to be published).

${ }^{14}$ P. Berger, K. Chang, P. Bhattacharya, J. Singh, and K. K. Bajaj, Appl. Phys. Lett. 53, 684 (1988).

${ }^{15}$ J. Pamulapati, R. Lai, G. I. Ng, Y. C. Chen, P. R. Berger, P. K. Bhattacharya, J. Singh, and D. Pavlidis, J. Appl. Phys. 68, 347 (1990). 